\title{
Rutinas profesionales de los becarios de Periodismo: el caso de Málaga
}

\author{
Kazetaritzako bekadunen lan-errutinak: \\ Malagako kasua
}

\section{Media routines of Journalism interns: the case of Málaga}

\section{Manuel García-Borrego' Sergio Roses Campos ${ }^{2}$}

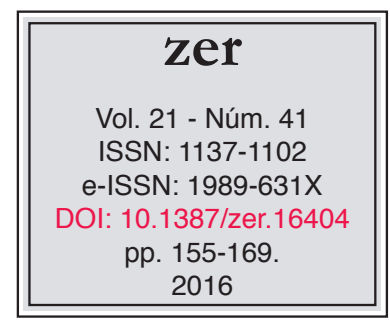

Recibido el 21 de enero de 2016, aceptado el 13 de mayo de 2016.

\section{Resumen}

Este artículo exploratorio describe las rutinas periodísticas de los becarios - fase crucial en la formación y socialización del profesional - de los principales medios de Málaga. Se suministró un cuestionario a 33 alumnos. Los resultados indican que dedican más de seis horas diarias tanto en la redacción como fuera, pues el teletrabajo está instaurado en la mayoría del colectivo, y elaboran unas tres piezas, generalmente multimedia. Asimismo, el $20 \%$ de los becarios no lee diariamente prensa y el $85 \%$ utiliza diariamente redes sociales con fines profesionales, lo cual indica una renovada praxis de los jóvenes periodistas con sus fuentes $\mathrm{y}$ audiencia.

Palabras clave: Rutinas periodísticas, jóvenes, formación, universidad, empresa.

\section{Laburpena}

Esplorazio-artikulu honek Malagako komunikabide nagusietako bekadunen kazetaritza-errutinak deskribatzen ditu; izan ere, bekadunen fasea osoa erabakigarria da lanbidearen inguruan trebatzeko eta sozializatzeko. Galdeketa bat egin zitzaien 33 ikasleri. Emaitzek adierazten dutenez, egunean sei ordu baino gehiago ematen dituzten hala erredakzioan nola kanpoan, telelana oso sartuta baitago kolektiboaren gehiengoan. Horrez gain, hiru pieza inguru egiten dituzte, oro har multimedia formatuan. Halaber, bekadunen $\% 20 \mathrm{k}$ ez dute prentsa egunero irakurtzen eta $\%$ 85ek egunero erabiltzen dituzte sare sozialak lanerako. Horrek esan nahi

\footnotetext{
Universidad de Málaga, manoletus@uma.es

2 Universidad de Málaga, sergioroses@uma.es
} 
du aldatu egin dela kazetari gazteek erabiltzen dituzten iturriak eta jendearengana iristeko erabiltzen dituzten baliabideak.

Gako-hitzak: Kazetaritza errutinak, gazteak, prestakuntza, unibertsitatea, enpresa.

\begin{abstract}
This exploratory article describes the media routines of interns - a crucial stage in the training and socialization of professionals - in the Málaga main media. A questionnaire was submitted to 33 interns. The results indicate that students spend over six daily hours on their professional activity, in the newsroom or out of it, as teleworking is widespread in the majority of the collective, and produce around three pieces, generally multimedia. Furthermore, $20 \%$ of the trainees don't read the newspaper daily and $85 \%$ use social networks for professional purposes, which indicates a renewed praxis in young journalists with their sources and audience.
\end{abstract}

Keywords: Journalistic routines, youth, training, university, company. 


\section{Introducción}

La realización de prácticas de empresa es una de las principales formas de acceso a la profesión y su importancia en términos cuantitativos ha crecido en los últimos años. La Asociación de la Prensa de Madrid encuestó entre 2006 y 2011 a los profesionales asociados al colectivo acerca de la manera en que accedieron a su puesto actual y mostró cómo el incremento de la estancia de becario como vía de inserción laboral se duplicó del 13,3\% en 2006 al 25,9\% en 2011 (APM, 2006-2011). Este fenómeno se produce en una etapa de formación especialmente delicada, en la que los estudiantes conforman su visión de la profesión (Pestano, Rodríguez-Wangüemert y Del Ponti, 2011) y van configurando su forma de trabajar a través de la socialización con los profesionales consolidados (Mellado et al., 2013), motivos que justifican el estudio de este fenómeno. El objetivo central de esta investigación ${ }^{3}$ estriba en describir las rutinas periodísticas de los becarios de los principales medios de Málaga, ciudad que sin ser capital regional presenta una amplia presencia de los grandes grupos nacionales y regionales de comunicación. Se trata, pues, de un escenario con características estructurales semejantes (e.g. tamaño de las redacciones) a la de la mayoría de los casos en que se produce el fenómeno estudiado en España. El estudio del caso de Málaga permite, por tanto, establecer una primera referencia sobre las rutinas profesionales de los estudiantes en prácticas, si bien los resultados no son extrapolables al resto del país.

\section{Marco teórico y estado de la cuestión}

De acuerdo con la literatura científica, las rutinas profesionales tienen un papel fundamental en la construcción de los contenidos periodísticos. El modelo de la jerarquía de influencias (Shoemaker y Reese, 1996; Reese, 1999, 2007) identifica cinco niveles interrelacionados que influyen en la producción de noticias: el individual, el de las rutinas mediáticas, el organizacional, el extramediático y el ideológico. Estas rutinas mediáticas incluyen, entre otras prácticas, el uso de fuentes, la organización de la información dentro del texto, las técnicas de construcción del relato o los propios hábitos de los redactores (Shoemaker y Reese, 1996).

Las rutinas profesionales serían, pues, siguiendo la definición aportada por los autores, una serie de prácticas y patrones que los trabajadores emplean para realizar sus tareas y que se repiten en el tiempo de manera 'rutinizada' (Shoemaker y Reese, 1996).

En el panorama español, destacamos la definición de Túñez (1999: 148): "pautas de comportamiento consolidadas en la profesión, asimiladas por costumbre y habitualmente ejecutadas de forma mecánica, que están presentes en todo el proceso de producción informativa". Una acotación posterior la amplía a la "autolimitación en la construcción del temario, el equilibrio percibido entre criterios periodísticos, políticos y económicos al seleccionar y jerarquizar las noticias, la iniciativa en las relaciones y la forma de contacto de los periodistas con sus fuentes, y los aspectos profesionales que se reconocen y valoran en las redacciones" (Túñez y MartínezSolana, 2014). Como se puede colegir, hasta el momento la literatura científica se ha centrado principalmente en la vertiente productiva de las rutinas periodísticas.

Agradecimientos: Universidad de Málaga. Programa de Becas de Iniciación a la Investigación 2015. 


\subsection{Un nuevo enfoque}

Esta investigación, sin embargo, plantea una reformulación del concepto de rutinas profesionales, al entender que entre ellas se encuentran tareas que no habían sido contempladas en la literatura previa, que ha situado su foco en lo que ocurre durante el horario de oficina. En nuestro caso, entendemos que junto a las rutinas de la producción de la noticia existen otras vinculadas estrechamente con la actividad profesional que, sin embargo, se producen al margen de las obligaciones laborales y contractuales, como sucede con la lectura de prensa, el consumo cultural o la interacción en redes sociales (ver Cuadro 1). Así, el periodista no lo sería solamente durante su jornada de trabajo, sino las 24 horas del día, ya que difícilmente un profesional puede ejercer adecuadamente su profesión estando desconectado del contexto informativo, social y cultural en que se halla.

Cuadro 1. Concepto de rutinas profesionales.

\begin{tabular}{|c|c|}
\hline En la literatura anterior & En este artículo \\
\hline $\begin{array}{c}\text { Foco en la actividad laboral (jornada } \\
\text { laboral) }\end{array}$ & Foco en la actividad profesional \\
(24 horas) \\
$\begin{array}{c}\text { Rutinas productivas } \\
\text { (newsmaking) }\end{array}$ & $\begin{array}{c}\text { Rutinas productivas } \\
\text { (newsmaking) }\end{array}$ \\
& $\begin{array}{c}\text { Rutinas de actualización informativa } \\
\text { (seguimiento medios) }\end{array}$ \\
& $\begin{array}{c}\text { Rutinas de reciclaje de conocimientos } \\
\text { (e.g. lectura de libros) } \\
\text { Rutinas de interacción social } \\
\text { (e.g. redes sociales) }\end{array}$ \\
\hline
\end{tabular}

\subsection{Las rutinas de los periodistas españoles}

Las rutinas productivas de los periodistas españoles se han visto afectadas por la convergencia digital (Masip, 2003; Díaz Noci et al., 2007), evolucionando así hacia un perfil más polivalente (Scolari et al., 2007). Uno de los trabajos más recientes y completos sobre rutinas profesionales es el de Túñez y Martínez Solana (2014), según el cual la jornada laboral promedia de los profesionales supera ligeramente las ocho horas y se extiende durante un promedio de 5,13 días semanales. Durante este horario, los periodistas confeccionan de media cinco piezas informativas, $\mathrm{y}$, además de redactar, el 58,4\% maqueta o edita sus propias piezas, el 32,3\% graba cortes de audio, el $28,2 \%$ toma las fotografías y hasta un $12,3 \%$ realiza vídeos. Todo ello coincide con las tendencias señaladas por Masip y Micó (2009): la adaptación a la era digital trae consigo una serie de nuevas rutinas profesionales que no necesariamente se traducen en una mejora de contenidos, ya que el aumento del número de tareas acaba redundando en un menor espacio de tiempo para realizar cada una de ellas.

Desafortunadamente, el número de estudios sobre las rutinas profesionales de los periodistas en prácticas es muy exiguo pese a la importancia de este periodo en la configuración de su cultura y formación profesional (García-Borrego, 2015). 
Lamuedra (2007) es la única en investigar sus rutinas dentro de la redacción. Desde una perspectiva eminentemente cualitativa, establece una distinción entre cuatro tipos diferentes de estudiantes según su rol en la empresa. El primer grupo, propio de medios digitales, "sólo manejó teletipos de agencias de noticias y su función consistió fundamentalmente en «cortar y pegar». En el segundo, repartido en todo tipo de medios, predominaba el manejo de "teletipos de agencias con el objeto de enriquecerlos y contrastarlos mediante llamadas telefónicas". El tercero, también frecuente en todas las plataformas, "salió a la calle, pero fundamentalmente a actos programados como ruedas de prensa". El cuarto y último grupo, mucho más reducido que los tres anteriores, "sí buscó temas propios con cierta asiduidad". Sucedería generalmente en periódicos locales durante la época estival, "cuando la agenda política pierde densidad y deja un vacío" informativo que rellenar.

Aunque no ejecutan un trabajo específico sobre el terreno y sitúan el principal foco en las condiciones laborales de las prácticas profesionales, la de Pérez-Serrano, Rodríguez-Barba y Rodríguez-Pallarés (2015) es la publicación más reciente en tocar las rutinas profesionales de los estudiantes. En su análisis de 1.396 ofertas de prácticas de la Universidad Complutense de Madrid dedican un breve capítulo a las aptitudes demandadas por las empresas. Su conclusión podría resumirse en que, a pesar de la pujanza de los perfiles polivalentes -en seis de las quince principales empresas se reconoce el desempeño de dos o más funciones-, "aún prima la especialización laboral frente al perfil profesional "todoterreno"” en los parámetros reseñados en la oferta (Pérez-Serrano, Rodríguez-Barba y Rodríguez-Pallarés, 2015).

\subsection{La enseñanza del periodismo en España}

Pese a esta prevalencia de los nuevos perfiles y la búsqueda de la especialización, Rosique (2013), como Sánchez-García (2016), concluye que la universidad no está siendo capaz de adaptarse a los cambios que surgen continuamente en la profesión $y$, aunque existen esfuerzos para reducir esta distancia, la falta de recursos hace que se tornen insuficientes. Para López (2012), es éste uno de los grandes retos de las facultades de comunicación: actualizar los contenidos para dar respuestas al entorno cambiante y proporcionar una formación más adecuada a los futuros profesionales.

La opinión de los estudiantes de Periodismo en universidades españolas se sitúa en esta misma línea. Humanes y Roses (2014) constatan que, según los alumnos encuestados, "la brecha que separa la formación universitaria del mundo profesional sigue siendo grande". Así, la teoría cuenta con un peso mayor en los planes de estudio en contraposición a la vertiente práctica del periodismo, poco atendida y alejada de la realidad actual de la profesión. En este contexto, la posibilidad de realizar prácticas en medios de comunicación confiere a este tipo de estancias un aliciente añadido para los periodistas en formación.

En el caso de Málaga, no existen datos exactos del número de periodistas que ejercen su labor, aunque sí se conoce que la FAPE (2016) cuenta con 261 asociados en la provincia. La Universidad de Málaga, la principal institución a la hora de nutrir a los medios de nuevos estudiantes de prácticas, produjo 124 egresados en 20132014, el último año del que existen datos disponibles (UMA, 2016). De ellos, menos de la mitad (un 46,16\%) se encuentra actualmente trabajando. Entre los egresados 
que han conseguido un primer empleo existe una tasa de temporalidad superior al $85 \%$, y en torno al $65 \%$ son jornadas a tiempo parcial (UMA, 2016).

\section{Hipótesis}

En base al estado actual de la literatura enunciamos las siguientes hipótesis:

H1. Las rutinas de producción de información de los estudiantes de prácticas tienden a asemejarse a las típicas de los profesionales consolidados por el proceso de socialización, aunque estas rutinas se desarrollan con menor intensidad dada la naturaleza formativa de su estancia en la empresa. Por esta razón, se espera que el número de piezas elaboradas diariamente o la diversidad de funciones desempeñadas sea inferior a la de los periodistas sénior.

H2. El consumo de información de los becarios es muy alto, en tanto que su nuevo rol profesional requiere de un dominio de la actualidad mayor al que estaban habituados durante sus estudios. La conexión con la actualidad debería ser constante y en cierto modo profunda. En este mismo sentido, se espera que los estudiantes utilicen otras vías secundarias para reforzar esa base amplia de conocimientos, como la lectura de libros u otro tipo de actividades de carácter cultural.

H3. El uso de redes sociales por parte de los becarios es muy alto, puesto que se trata de una generación joven más familiarizada con las nuevas tecnologías a la que no le supone un esfuerzo añadido valerse de ellas para acceder a fuentes, encontrar nuevos temas sobre los que trabajar o compartir sus artículos.

\section{Metodología}

La población de estudio la constituyen los estudiantes universitarios que desarrollan sus prácticas profesionales en los principales medios de comunicación de Málaga y que realizan tareas de producción de contenidos, excluyendo así tanto a los fotógrafos como a aquéllos dedicados a la edición o la maquetación. Estas prácticas pueden ser curriculares o extracurriculares, es decir, podrán llevar o no consigo una compensación económica por el trabajo realizado. El estudio se circunscribe a Málaga por varios motivos: se trata de la sexta ciudad española por población en 2015; entre su ecosistema mediático se encuentran importantes grupos de comunicación nacionales y regionales (Vocento, Prensa Ibérica, PRISA, Unidad Editorial, Joly, Atresmedia); el tamaño de las redacciones es similar al de otras capitales autonómicas y de provincia en España; y el rol de la Universidad de Málaga -junto con la de Sevilla la única de titularidad pública que imparte el Grado en Periodismo en Andalucía-, cuya oferta de prácticas curriculares y extracurriculares es abundante. 
Esta primera aproximación exploratoria propone un diseño muestral no probabilístico que combina criterios estratégicos y de conveniencia de acuerdo con los estándares habituales para estudios exploratorios.

Los puntos de muestreo los componen los nueve principales medios y agencias de Málaga según parámetros como la difusión ${ }^{4}$, la audiencia ${ }^{5}$ y las visitas ${ }^{6}$, garantizando la diversidad en el soporte, el tamaño y la propiedad de los medios estudiados. Siguiendo el ejemplo de Lamuedra (2007), no se mencionan empresas ni nombres propios para salvaguardar la confidencialidad de unos y otros. En cualquier caso, resulta pertinente mencionar que entre los puntos de muestreo había cuatro periódicos, tres radios y dos agencias de noticias, y que los nueve medios utilizados eran de tirada nacional o locales pertenecientes a grupos mediáticos estatales o regionales. Las principales emisoras de televisión nacionales que operan en Málaga quedaron excluidas al no disponer de estudiantes durante el período en que se lanzó la encuesta, lo cual supone hasta cierto punto una limitación en el estudio.

En suma, la población se compone de 38 estudiantes en prácticas. Se suministró la encuesta al total los estudiantes para obtener el mayor número de respuestas. Finalmente se recibieron 33 respuestas, lo cual supone un $87 \%$ del total de estudiantes inicialmente contemplados. Las características de la muestra pueden consultarse en la Tabla 1.

Tabla 1. Características de la muestra.

\begin{tabular}{|c|c|}
\hline Mujer & $39,4 \%(13 \text { de } 33)^{1}$ \\
\hline Edad & $M=23 \mathrm{DT}=2,136$ \\
\hline $\begin{array}{l}\text { Han completado el } 75 \% \text { o más de los } \\
\text { créditos del Grado }\end{array}$ & $81,8 \%$ (27 de 33$)$ \\
\hline $\begin{array}{l}\text { Pertenece a la Universidad de } \\
\text { Málaga }\end{array}$ & $75,8 \%$ (25 de 33$)$ \\
\hline $\begin{array}{l}\text { Recibe retribución (prácticas } \\
\text { extracurriculares) }\end{array}$ & Sí= 93,9\% (31 de 33) \\
\hline Experiencia total en prácticas & Más de seis meses= $48,5 \%(16$ de 33$)$ \\
\hline Medio & $\begin{array}{l}\text { Prensa }=57,6 \% \text { ( } 19 \text { casos) } \mid \text { Radio }=24,2 \% \text { (8 } \\
\text { casos) } \mid \text { Agencias }=18,2 \% \text { ( } 6 \text { casos })\end{array}$ \\
\hline Trabaja en medio privado & $90,9 \%$ (30 de 33$)$ \\
\hline Trabaja en una redacción ${ }^{2}$ & $\begin{array}{l}\text { Pequeña }=48,5 \% \text { ( } 16 \text { casos) | Mediana }=33,3 \% \\
\text { (11 casos) | Grande }=18,2 \% \text { (6 casos) }\end{array}$ \\
\hline
\end{tabular}

El cuestionario, compuesto por 21 preguntas (ver Anexo), se suministró por vía digital-generalmente por correo electrónico- y se respondió entre los días 3 y 14 de

\footnotetext{
${ }^{4}$ OJD (Octubre 2015) - Totales - Tráfico Nacional e Internacional. http://www.ojdinteractiva.es/medios-digitales

5 AIMC. Estudio General de Medios (Octubre de 2014 a Mayo de 2015). http://www.aimc.es/Entregade-resultados-EGM-1 ${ }^{\text {a }}$-ola,1588.html

${ }^{6}$ comScore. Top España Enero 2015.
} 
agosto de 2015, tratando así de que aquellos estudiantes que se incorporaron a sus puestos a principios del mes de julio gozaran del tiempo suficiente para responder con criterio y fidelidad a las preguntas formuladas. Previamente, el cuestionario había sido sometido al juicio de un focus group de actuales y anteriores becarios.

Las variables estudiadas, con las que se pretende abarcar toda la actividad profesional que puede redundar en el día a día del estudiante, se subdividen en cuatro grupos:

Las rutinas de producción, siguiendo el concepto clásico que comprende el número de piezas elaboradas, el tiempo disponible para cada una de ellas o las funciones asumidas además de redactar.

Las rutinas de actualización informativas, entendidas como el consumo puro de información, ya sea generalista o especializada, a través de medios de comunicación.

El reciclaje de conocimientos, es decir, el acceso a aquellas fuentes de conocimientos secundarias como la lectura de libros, el visionado de películas, series o contenidos audiovisuales u otras actividades de carácter cultural.

Las rutinas de interacción social, que miden la frecuencia de acceso a las redes sociales Facebook y Twitter y el tipo de uso que se les da a éstas (profesional o personal) tanto en el lugar de trabajo como fuera de él.

La operacionalización de las variables (ver Anexo) relacionadas con las rutinas productivas se fundamentó en las empleadas por la APM (2004-2014), RodríguezAndrés (2003), Díaz Noci et al. (2007), Túñez (2009) o Túñez y Martínez-Solana (2014), entre otros. Las rutinas de actualización informativa y reciclaje de conocimientos se operacionalizaron según el modelo del CIS (1999, 2003, 2010, 2013 y 2014), el EGM o Casero-Ripollés (2012), así como los ya mencionados de la APM (2004-2014). Igualmente, se crearon ítems ad hoc para medir variables específicas como sucede en el caso de la interacción social, operacionalizada a partir. El cuestionario también recogió información sociodemográfica de los participantes.

\section{Resultados}

\section{1. Newsmaking}

La duración media de la jornada laboral es de 6,61 horas (DT=1,20). Del total de tiempo en la oficina, la mayoría se emplea en producir los contenidos del día (16 de los 33, un $48,5 \%$, destinan más de dos horas de su jornada), si bien al 21,2\% (siete casos) apenas le lleva entre media hora y una hora. Más de la mitad de los estudiantes (17 de los 33) destina menos de treinta minutos a recabar información sobre sus temas, prefiriéndose como apoyo para las piezas el contacto con las fuentes personales (en torno al 60\% dedica más de 30 minutos diarios en establecer contacto con informantes para sus artículos).

El segundo lugar donde emplean más tiempo los alumnos es en la calle: 19 de los 33 (un 57,6\%) pasan al menos una hora trabajando fuera de la redacción, de los cuales un cuarto dedica incluso más de dos horas.

La mayoría $(53,1 \%)$ de los estudiantes de Periodismo que realizan prácticas en medios de comunicación confeccionan tres o más piezas al día, llevando la cifra promedia a 2,72 con una desviación típica de 1,276. Todos los estudiantes cubren al menos una noticia al día y se dan cuatro casos de jóvenes que escriben cinco o más piezas en cada jornada (ver Gráfico 1). 
Gráfico 1. Número de piezas diarias realizadas por los estudiantes de prácticas.

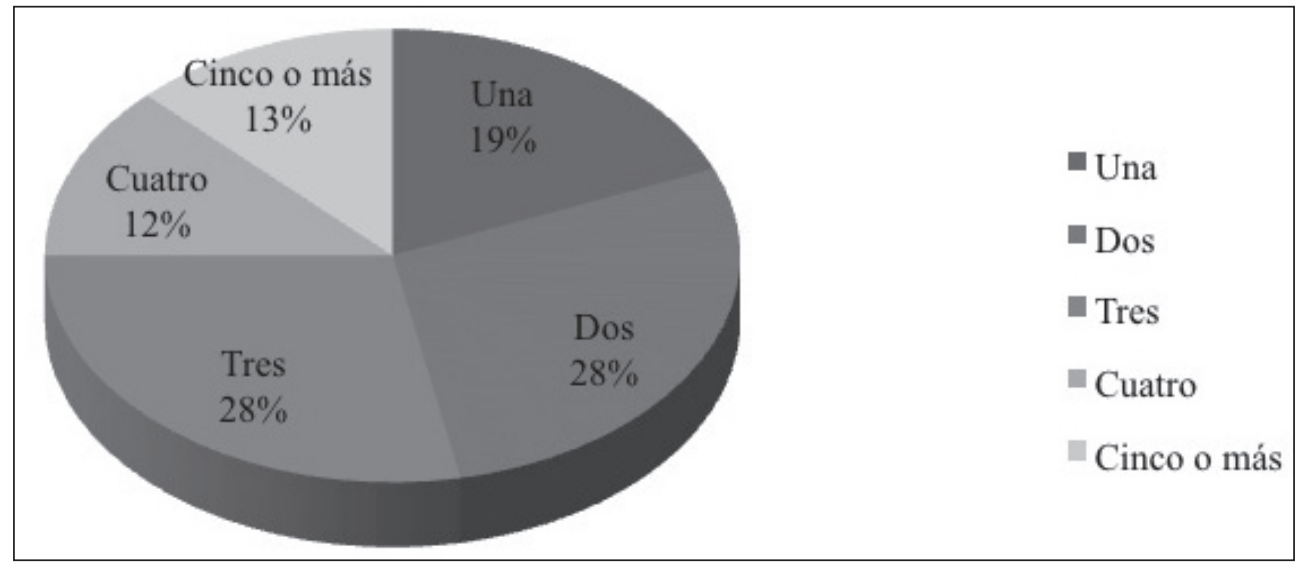

Fuente: Elaboración propia.

El tiempo dedicado a elaborar cada una de estas piezas varía indistintamente entre los grupos, aunque resulta llamativo que 15 estudiantes (un 45,3\% del total) gozan de menos de una hora para desarrollar cada una de ellas -de los cuales, además, un tercio cuenta con menos de 30 minutos-.

Sobre la multidisciplinariedad del periodista, resulta destacable que casi tres cuartas partes de los estudiantes ( 24 casos) toma fotos además de redactar los textos. A esto se añade que el 57,6\% realiza grabaciones de audio, el 15,2\% de vídeo y que el $51,5 \%$ edita o maqueta sus propios contenidos. Entre el resto de funciones extra destacadas por otros estudiantes sobresalen los trabajos administrativos, la elaboración de infografías, la gestión de las redes sociales o la organización y la actualización de la web del medio. El 100\% de los estudiantes desempeña alguna función complementaria a la redacción.

En lo referente a los lugares o dispositivos desde los que los estudiantes en prácticas han escrito o elaborado sus piezas alguna vez, destaca que prácticamente la mitad de los becarios consultados (16 de 33), ha escrito alguna vez desde su propia casa. Una cifra similar de alumnos ha enviado y producido sus contenidos para el medio donde trabaja desde su terminal móvil (hasta 13, un 39,4\% del total) y desde la calle (12 casos, 37,4\%). Por último, cinco estudiantes reconocieron haber hecho uso de dispositivos móviles u ordenadores de otras personas para redactar sus temas. En general, aproximadamente dos de cada tres estudiantes (21 de los 33) ha debido escribir en algún momento desde fuera de las oficinas del medio donde trabaja.

De hecho, al consultarles sobre el tiempo empleado en tareas relacionadas con su trabajo (preparación de temas, búsqueda de ideas, etcétera) tras acabar la jornada presencial o durante los días libres, se encontró que sólo cinco estudiantes encuestados pueden desconectar completamente del trabajo una vez en casa. De los 28 casos restantes (un $85 \%$ del total), la mitad dedica más de 30 minutos a sus labores y cinco amplían su jornada en más de una hora. A pesar de que esta situación sucede con menos frecuencia en los días de descanso, más de la mitad de los estudiantes (19, un 
$57,6 \%$ ) continúa vinculado a su trabajo en las fechas libres, y aunque la mayoría de ellos no dedique más de media hora, se dan determinados casos en los que se supera la hora e incluso las dos horas.

\subsection{Actualización informativa}

Hasta 26 de los 33 estudiantes que realizan prácticas (78,8\% del total) afirman que repasan la prensa todos o casi todos los días, lo cual indica que alrededor de uno de cada cinco alumnos no lee el periódico a diario. El consumo de informativos en radio o televisión es ligeramente inferior (el 60,6\% lo hace todos o casi todos los días), mientras que los programas de tienen un consumo más minoritario (ver Tabla 2).

Tabla 2. Frecuencia de actualización informativa de los becarios.

\begin{tabular}{|l|c|c|c|c|c|}
\hline \multicolumn{1}{|c|}{ FRECUENCIA } & $\begin{array}{c}\text { Todos } \\
\text { o casi } \\
\text { todos } \\
\text { los días }\end{array}$ & $\begin{array}{c}\text { Cuatro o } \\
\text { cinco días } \\
\text { por semana }\end{array}$ & $\begin{array}{c}\text { Dos o tres } \\
\text { veces por } \\
\text { semana }\end{array}$ & $\begin{array}{c}\text { Una vez a } \\
\text { la semana }\end{array}$ & $\begin{array}{c}\text { Con menor } \\
\text { frecuencia }\end{array}$ \\
\hline $\begin{array}{l}\text { Prensa (impresa } \\
\text { o digital) }\end{array}$ & $78,8 \%$ & $15,2 \%$ & $6,1 \%$ & $0,0 \%$ & $0,0 \%$ \\
\hline $\begin{array}{l}\text { Informativos } \\
\text { (radio/TV) }\end{array}$ & $60,6 \%$ & $21,2 \%$ & $18,2 \%$ & $0,0 \%$ & $0,0 \%$ \\
\hline $\begin{array}{l}\text { Programas de } \\
\text { debate (radio/TV) }\end{array}$ & $9,1 \%$ & $9,1 \%$ & $45,5 \%$ & $12,1 \%$ & $24,2 \%$ \\
\hline
\end{tabular}

Si se toma como unidad de tiempo el día para analizar el consumo de estos formatos, y distinguiendo a su vez las rutinas desarrolladas durante el horario de trabajo, fuera de él y en los días de descanso, se aprecian datos de interés como que más de la mitad de los estudiantes (18 casos de 33) lee la prensa menos de media hora durante la jornada laboral (ver Tabla 3). Fuera del trabajo, 27 de los encuestados (un 81,8\%) dedican menos de una hora a la lectura -la mitad de los cuales no destina más de media hora- y dos no dedican nada. La mayoría de los becarios (19) no llega a la hora de lectura diaria.

En las jornadas de descanso, la gran mayoría de los estudiantes (28 de los 33 casos, un $84,4 \%$ ) afirma leer los periódicos por un tiempo menor a una hora, con cinco casos que optan por la desconexión total y no hacen ningún tipo de consumo y otros 12 (un 36,4\% del total) que emplea menos de media hora diaria. En resumen, la mayoría $(51,5 \%)$ acaba leyendo menos de 30 minutos.

El consumo de informativos se mantiene estable, aunque es obviamente inferior en las oficinas del medio. El consumo de información especializada es menor que el de prensa generalista en horario de oficina - uno de cada tres becarios no acude a este tipo de contenidos dentro de la redacción-; no obstante, es el preferido tanto al acabar la jornada como en los días de descanso. Sólo cuatro estudiantes aseguran no consumir ningún tipo de producto temático. 
Tabla 3. Tiempo diario dedicado a la actualización informativa.

\begin{tabular}{|l|c|c|c|c|c|c|c|c|c|}
\hline \multirow{2}{*}{} & \multicolumn{3}{|c|}{ Trabajo (Redacción) } & \multicolumn{3}{c|}{ Trabajo (Casa) } & \multicolumn{3}{c|}{ Descanso (todo el día) } \\
\cline { 2 - 10 } & $\mathbf{0 - 3 0 m}$ & $\mathbf{3 1 \mathbf { m } - 1 \mathbf { h }}$ & $\mathbf{> 1 \mathbf { h }}$ & $\mathbf{0 - 3 0 m}$ & $\mathbf{3 1 \mathbf { m } - 1 \mathbf { h }}$ & Más 1h & $\mathbf{0 - 3 0 m}$ & $\mathbf{3 1 m - 1 h}$ & $>\mathbf{1 h}$ \\
\hline Prensa & $54,5 \%$ & $27,3 \%$ & $18,2 \%$ & $48,5 \%$ & $39,4 \%$ & $12,1 \%$ & $51,5 \%$ & $33,3 \%$ & $12,1 \%$ \\
\hline Inform. & $\mathbf{8 4 , 8} \%$ & $6,1 \%$ & $9,1 \%$ & $48,5 \%$ & $42,4 \%$ & $9,1 \%$ & $45,5 \%$ & $39,4 \%$ & $12,1 \%$ \\
\hline Debate & $90,9 \%$ & $3,0 \%$ & $3,0 \%$ & $69,7 \%$ & $24,2 \%$ & $6,1 \%$ & $72,7 \%$ & $18,2 \%$ & $6,1 \%$ \\
\hline Especial. & $72,7 \%$ & $15,2 \%$ & $12,1 \%$ & $48,5 \%$ & $27,3 \%$ & $24,2 \%$ & $42,4 \%$ & $33,3 \%$ & $21,2 \%$ \\
\hline
\end{tabular}

Fuente: Elaboración propia.

\subsection{Reciclaje de Conocimientos}

La lectura de libros está repartida en grupos diferenciados, si bien tres de cada cuatro afirma leer al menos una vez a la semana, con un sector del 36,4\% (12 casos) que declara acudir a los libros cuatro o más veces por semana (ver Tabla 4). El 27,3\% (nueve casos) lee con una frecuencia menor a un día a la semana.

Tabla 4. Frecuencia del reciclaje de conocimientos.

\begin{tabular}{|l|c|c|c|c|c|}
\hline FRECUENCIA & $\begin{array}{c}\text { Todos } \\
\text { o casi } \\
\text { todos } \\
\text { los días }\end{array}$ & $\begin{array}{c}\text { Cuatro } \\
\text { o cinco } \\
\text { días por } \\
\text { semana }\end{array}$ & $\begin{array}{c}\text { Dos o tres } \\
\text { veces por } \\
\text { semana }\end{array}$ & $\begin{array}{c}\text { Una vez a } \\
\text { la semana }\end{array}$ & $\begin{array}{c}\text { Con menor } \\
\text { frecuencia }\end{array}$ \\
\hline $\begin{array}{l}\text { Programas culturales/ } \\
\text { divulgativos/documental }\end{array}$ & $3,0 \%$ & $6,1 \%$ & $27,3 \%$ & $39,3 \%$ & $24,2 \%$ \\
\hline Lectura de libros & $21,2 \%$ & $15,2 \%$ & $15,2 \%$ & $21,2 \%$ & $27,3 \%$ \\
\hline
\end{tabular}

Fuente: Elaboración propia.

Los documentales y programas culturales o divulgativos son los siguientes en preferencia según los estudiantes, si bien aproximadamente uno de cada cuatro (nueve casos) no visiona este tipo de contenidos en sus momentos libres.

Por último, el consumo de libros sería el que recibe mayor dedicación, con un $30,3 \%$ de los estudiantes (diez casos) que afirma leer más de una hora en los días que no deben acudir a la oficina, pese a que el $36,4 \%$ ( 12 casos) dedica entre 0 y 30 minutos (ver Tabla 5).

Tabla 5. Tiempo diario dedicado al reciclaje de conocimientos.

\begin{tabular}{|l|c|c|c|c|c|c|c|c|c|}
\hline & \multicolumn{3}{|c|}{ Trabajo (Redacción) } & \multicolumn{3}{c|}{ Trabajo (Casa) } & \multicolumn{3}{c|}{ Descanso (todo día) } \\
\cline { 2 - 10 } & $\mathbf{0 - 3 0 m}$ & $\mathbf{3 1 m - 1 h}$ & $\mathbf{> 1 h}$ & $\mathbf{0 - 3 0 m}$ & $\mathbf{3 1 m - 1 h}$ & Más 1h & $\mathbf{0 - 3 0 m}$ & $\mathbf{3 1 m - 1 h}$ & $>\mathbf{1 h}$ \\
\hline Prog. cultural & $90,9 \%$ & $6,1 \%$ & $3,0 \%$ & $69,7 \%$ & $24,2 \%$ & $6,1 \%$ & $66,7 \%$ & $18,2 \%$ & $9,1 \%$ \\
\hline Libros & $97,0 \%$ & $0 \%$ & $3,0 \%$ & $48,5 \%$ & $30,3 \%$ & $21,2 \%$ & $36,4 \%$ & $30,3 \%$ & $30,3 \%$ \\
\hline
\end{tabular}

Fuente: Elaboración propia. 


\subsection{Interacción Social}

Mención aparte merece el uso de Facebook y Twitter, las dos grandes redes sociales, tanto en el uso profesional como en el meramente personal y de ocio, interesante para establecer la comparativa.

El 72,7\% (24 casos) de los estudiantes de Periodismo usa Facebook dentro de la redacción. Resulta llamativo que más de la mitad de los becarios (18 de los 33) lo haga por motivos no profesionales (ver Tabla 6). Se trata prácticamente del mismo número de estudiantes que accede para contactar con fuentes, recabar opiniones u otras funciones periodísticas, con lo cual puede pensarse que en una conexión habitual se desarrollan ambas acciones. El uso de Facebook por razones laborales se extiende también a los momentos libres de un día normal de trabajo, pues ya en casa el 19 de los 33 estudiantes emplean tiempo en este tipo de cometidos. De hecho, lo hacen incluso con mayor frecuencia que en la redacción. Hasta en los días libres, nueve de los alumnos encuestados (un 27,3\%) utilizan la red social por motivos laborales.

La presencia de Twitter en las redacciones es mucho mayor tanto por razones de trabajo (84,8\% de uso entre los estudiantes) como por motivos personales o de ocio (63,6\% de acceso en la sede de la empresa). El uso de Twitter con una finalidad profesional se prolonga hasta las horas fuera del puesto de trabajo (así es en 15 de los 33 casos). En días de descanso, uno de cada tres sigue conectado con su empresa a través de esta red social, con casos concretos que, como con Facebook, llegan a superar la hora.

Tabla 6. Interacción social diaria.

\begin{tabular}{|l|c|c|c|c|c|c|c|c|c|}
\hline \multirow{2}{*}{} & \multicolumn{3}{|c|}{ Trabajo (Redacción) } & \multicolumn{3}{c|}{ Trabajo (Casa) } & \multicolumn{3}{c|}{ Descanso (todo día) } \\
\cline { 2 - 10 } & $\mathbf{0 m}$ & $\mathbf{1 m - 3 0 m}$ & $\begin{array}{c}\text { Más } \\
\mathbf{3 0 m}\end{array}$ & $\mathbf{0 m}$ & $\mathbf{1 m - 3 0 m}$ & $\begin{array}{c}\text { Más } \\
\mathbf{3 0 m}\end{array}$ & $\mathbf{0 m}$ & $\mathbf{1 m - 3 0 m}$ & $\begin{array}{c}\text { Más } \\
\mathbf{3 0 m}\end{array}$ \\
\hline FB $\boldsymbol{t r}^{\mathbf{3}}$ & $42,4 \%$ & $45,5 \%$ & $12,1 \%$ & $42,4 \%$ & $30,3 \%$ & $27,3 \%$ & $72,7 \%$ & $18,2 \%$ & $6,1 \%$ \\
\hline FB p/o & $45,5 \%$ & $45,5 \%$ & $9,1 \%$ & $6,1 \%$ & $30,3 \%$ & $60,6 \%$ & $0,0 \%$ & $39,4 \%$ & $57,6 \%$ \\
\hline Tw tr & $15,2 \%$ & $45,5 \%$ & $39,4 \%$ & $54,5 \%$ & $18,2 \%$ & $27,3 \%$ & $66,7 \%$ & $24,2 \%$ & $6,1 \%$ \\
\hline Tw p/o & $36,4 \%$ & $45,5 \%$ & $18,2 \%$ & $24,2 \%$ & $30,3 \%$ & $42,4 \%$ & $15,2 \%$ & $36,4 \%$ & $45,5 \%$ \\
\hline
\end{tabular}

Fuente: Elaboración propia.

\section{Conclusiones}

Antes de enunciar las conclusiones más relevantes, resulta necesario recalcar el alcance de la investigación. Al tratarse de un estudio exploratorio, no es posible generalizar los resultados al conjunto de los becarios españoles. Sin embargo, la escasez de datos en la literatura sobre el fenómeno de estudio otorga relevancia a los mismos, pues establecen las primeras referencias sobre las rutinas profesionales de los becarios, entendidas éstas desde un punto de vista amplio: incorporando al núcleo clásico de producción de noticias otras rutinas como son la actualización informativa, el reciclaje de conocimientos y la interacción social. 
Las rutinas profesionales de los becarios del estudio vinculadas a la producción de noticias demuestran ser ciertamente intensas, más de lo contemplado en primera instancia en la $\mathrm{H} 1$, si bien no parecen llegar a los niveles de los profesionales consolidados. Más de la mitad de los estudiantes reconocen confeccionar diariamente tres o más piezas, unas cifras cercanas a las proporcionadas por Túñez y Martínez Solana (2014). Asimismo, en lo referente a la diversidad de tareas desempeñadas, los datos extraídos indican que la fotografía, la grabación de audios y vídeos y la edición y maquetación están incluso mucho más extendidas entre los becarios que entre los profesionales sondeados por Túñez y Martínez Solana (2014). Por lo tanto, la polivalencia, sumada al teletrabajo y al uso redaccional del teléfono móvil, parece ser un rasgo aún más acentuado en las nuevas generaciones de periodistas. Esta acumulación de funciones podría indicar un incremento de la importancia de los estudiantes en las redacciones, por lo que surge una línea de investigación de interés en el estudio de las condiciones exactas en las que se desarrollan las prácticas profesionales en empresas periodísticas.

Por otra parte, alrededor de un $80 \%$ de los becarios del estudio sigue la actualidad informativa a través de la prensa con una periodicidad diaria. Si bien este porcentaje es bastante alto en comparación con la población general de su misma edad -el $27,7 \%$ lee prensa diariamente (CIS, 2015)-, su condición de periodistas en formación obliga a considerar estos valores insuficientes. Por tanto, la H2 queda refutada. El seguimiento de la prensa es una práctica fundamental en el ejercicio periodístico tanto para estar al tanto de lo que ocurre como para conocer el trabajo de otros profesionales en las áreas informativas que son de su responsabilidad. La actualización informativa parece ser una rutina que, aunque también se extienda al tiempo libre, se produce principalmente en la redacción. Por lo general, en los momentos o jornadas de descanso la preferencia es la del consumo de información más especializada (deportes, música...).

Alrededor de una cuarta parte de los periodistas en prácticas apenas lee libros. De nuevo los resultados contradicen la $\mathrm{H} 2$, y nos encontramos con unos datos que deben llevar a la reflexión. Una explicación del fenómeno podría encontrarse en la percepción estudiantil de que, si bien la actualización informativa produce unos efectos inmediatos en el desempeño de la labor periodística, la formación de un fondo cultural amplio implica un proceso a más largo plazo cuyos resultados son menos visibles en el día a día. Por otra parte, hablamos de una generación de nativos digitales que probablemente utiliza otras vías como internet para reciclar sus conocimientos. Futuros trabajos deberían indagar en este sentido.

Por último, las magnitudes de las variables referidas a la interacción social con el entorno a través de las herramientas digitales indican en los periodistas de esta generación una renovada praxis de las relaciones sociales con sus fuentes, su audiencia y el resto de la sociedad que tiene como base las conexiones virtuales vehiculadas por las redes sociales. Así, la H3 queda apoyada por los datos.

Los datos exploratorios proporcionados en esta investigación abren la puerta a futuros estudios sobre cómo se conforman las rutinas profesionales de los periodistas durante el periodo de formación en prácticas. Las próximas investigaciones deberán, primero, servirse de muestras que permitan ofrecer datos descriptivos más generalizables y, segundo, examinar la posible impronta en las rutinas de los becarios de variables 
vinculadas con el nivel organizativo de los medios (tamaño de la redacción, carga de trabajo del resto de periodistas, nivel de convergencia multimedia), su titularidad (pública o privada), su ubicación en Madrid o Barcelona frente al resto de territorios.

\section{Referencias bibliográficas}

APM (2004-2014). Informe Anual de la Profesión Periodística. Madrid: Asociación de la Prensa de Madrid.

CASERO-RIPOLLÉS, A. (2012). Más allá de los diarios: el consumo de noticias de los jóvenes en la era digital. Comunicar, 39, 151-158. http://dx.doi.org/10.3916/ C39-2012-03-05

CIS (2014). Barómetro de diciembre de 2014. http://www.cis.es/cis/opencms/-Archivos/Marginales/3040_3059/3047/cru3047edad.html

DÍAZ NOCI, J.; MESO, K.; LARRONDO, A.; SALAVERRÍA, R.; y SADABA, M. R. (2007). Presencia y uso de Internet en las redacciones de los diarios regionales vascos y navarros. Euskonews \& Media, 384. http://www.euskonews. com/0383zbk/gaia38301es.html

FAPE (2016). Periodistas, 40. http://fape.es/wp-content/uploads/2016/04/Periosdistas40.pdf

GARCÍA-BORREGO, M. (2015). Los estudiantes en prácticas en los medios españoles: una revisión bibliográfica. Revista Opción, 31(4), 481-498. http://produccioncientificaluz.org/index.php/opcion/article/view/20565/20476

HUMANES, M. L. y ROSES, S. (2014). Valoración de los estudiantes sobre la enseñanza del Periodismo en España. Comunicar, 42, 181-188. http://www.revistacomunicar.com/verpdf.php?numero=42\&articulo=42-2014-18

LAMUEDRA, M. (2007). Estudiantes de Periodismo y prácticas profesionales: el reto del aprendizaje. Comunicar, 28, 203-211. http://bit.ly/1ikfNY6

LÓPEZ, X. (2012). La formación de los periodistas para los entornos digitales actuales. Revista de Comunicación, 11, 178-195.

MASIP, P. (2003). Presencia y uso de Internet en las redacciones catalanas. Zer, Revista de Estudios de Comunicación, 14. http://www.ehu.eus/zer/hemeroteca/pdfs/ zer14-02-masip.pdf

MASIP, P. y MICÓ-SANZ, J. L. (2009). El periodista polivalente en el marco de la convergencia empresarial. Quaderns del CAC, 31-32, 91-99. https://www.cac. cat/pfw_files/cma/recerca/quaderns_cac/Q31-32_Masip_ES.pdf

MELLADO, C.; HANUSCH, F.; HUMANES, M. L.; ROSES, S.; PEREIRA, F.; YEZ, L.; DE-LEÓN, S.; MÁRQUEZ, M.; SUBERVI, F.; WYSS, V. (2013). The pre-socialization of future journalists. Journalism Studies, 14(6), 857-874. http:// dx.doi.org/10.1080/1461670X.2012.746006

PÉREZ-SERRANO, M. J.; RODRÍGUEZ-BARBA, D. y RODRÍGUEZ-PALLARES, M. (2015). Mercado de la Comunicación y estudiantes de Periodismo. 
Estructura de la demanda de perfiles profesionales. Revista Latina de Comunicación Social, 70, 209-229. http://dx.doi.org/10.4185/RLCS-2015-1043

PESTANO, J. M.; RODRÍGUEZ WANGÜEMERT, C.; y DEL PONTI, P. (2011). Transformaciones en los modelos de formación de periodistas en España. El reto europeo. Estudios sobre el mensaje periodístico, 17(2), 401-415. http://dx.doi. org/10.5209/rev_ESMP.2011.v17.n2.38122

REESE, S. (1999). Hacia una comprensión del periodista global. El modelo de investigación de ‘jerarquía de influencias'. Comunicación y Sociedad, 12, 47-68. http://www.unav.es/fcom/comunicacionysociedad/es/articulo.php?art_id=131

REESE, S. (2007). "Journalism research and the hierarchy of influences model: a global perspective”. Brazilian Journalism Research, 3, 29-42. http://bit.ly/1JBp2K2

ROSIQUE, G. (2013). Los estudios universitarios de periodismo en España: la adaptación al EEES y la formación de periodistas en competencias digitales. Revista Internacional de Estudios sobre Sistemas Educativos, 1(1-2), 117-132. http:// www.academia.edu/24153840/Los_estudios_universitarios_de_periodismo_en_ España_la_adaptación_al_EEES_y_la_formación_de_periodistas_en_competencias_digitales

RODRÍGUEZ-ANDRÉS, R. (2003). Características socio-demográficas y laborales de los periodistas españoles e índice de satisfacción profesional. Ámbitos, Revista Internacional de Comunicación, 9-10. http:/grupo.us.es/grehcco/ambitos09-10/ rodriguez.pdf

SÁNCHEZ-GARCÍA, Pilar (2016). Los efectos de la primera fase del EEES en la enseñanza del periodismo en España: mayor especialización y formación práctica. Communication \& Society, 29(1), 125-143. http://www.unav.es/fcom/communication-society/descarga_doc.php?art_id=564

SCOLARI, C.A.; Micó-Sanz, J. L.; Navarro-Guere, H.; Pardo-KuklisnkI, H. (2007). El periodista polivalente. Transformaciones en el perfil del periodista a partir de la digitalización de los medios audiovisuales catalanes. Zer, Revista de Estudios de Comunicación, 25, 37-60. http://www.ehu.es/zer/hemeroteca/pdfs/zer2502-scolari.pdf

SHOEMAKER, P. y REESE, S. (1996). Mediating the message: theories of influences on mass media content. Nueva York: Longman. https://journalism.utexas.edu/ sites/journalism.utexas.edu/files/attachments/reese/mediating-the-message.pdf

TÚÑEZ, M. (1999). Producir noticias. Cómo se fabrica la realidad periodística. Santiago de Compostela: Tórculo.

TÚÑEZ, M. (2009). Jóvenes y prensa en papel en la era Internet. Estudio de hábitos de lectura, criterios de jerarquía de noticias, satisfacción con los contenidos informativos y ausencias temáticas. Estudios sobre el Mensaje Periodístico, 15, 503-524. http://revistas.ucm.es/index.php/ESMP/article/view/ESMP0909110503A/11761

TÚNEZZ, M. y MARTÍNEZ-SOLANA, M. Y. (2014). Análisis del impacto de la función, las actitudes y las condiciones laborales del periodista en la producción de noticias: Hacia un periodismo de empresa. Zer, Revista de Estudios de Comunicación, 19(36), 169-182. http://www.ehu.eus/ojs/index.php/Zer/article/ view/13486/12072 